\title{
Finite Element Simulation of Reference Point Indentation on Bone
}

\author{
Ashraf Idkaidek, Vineet Agarwal and Iwona Jasiuk ${ }^{*}$ \\ Department of Mechanical Science and Engineering \\ University of Illinois at Urbana-Champaign \\ 1206 West Green Street, Urbana, IL 61801
}

${ }^{*}$ Corresponding author

Phone: (217) 333-9259

E-mail: ijasiuk@illinois.edu 


\section{Abstract}

Reference Point Indentation (RPI) is a novel technique aimed to assess bone quality. Measurements are recorded by the BioDent instrument that applies multiple indents to the same location of cortical bone. Ten RPI parameters are obtained from the resulting force-displacement curves. Using the commercial finite element analysis software Abaqus, we assess the significance of the RPI parameters. We create an axisymmetric model and employ an isotropic viscoelastic-plastic constitutive relation with damage to simulate indentations on a human cortical bone. Fracture of bone tissue is not simulated for simplicity. The $\mathrm{RPI}$ outputs are computed for different simulated test cases and then compared with experimental results, measured using the BioDent, found in literature. The number of cycles, maximum indentation load, indenter tip radius, and the mechanical properties of bone: Young's modulus, compressive yield stress, and viscosity and damage constants, are varied. The trends in the RPI parameters are then investigated.

We find that the RPI parameters are sensitive to the mechanical properties of bone. An increase in Young's modulus of bone causes the force-displacement loading and unloading slopes to increase and the total indentation distance (TID) to decrease. The compressive yield stress is inversely proportional to a creep indentation distance (CID1) and the TID. The viscosity constant is proportional to the CID1 and an average of the energy dissipated (AvED). The maximum indentation load is proportional to the TID, CID1, loading and unloading slopes, and AvED. The damage parameter is proportional to the TID, but it is inversely proportional to both the loading and unloading slopes and the AvED. The value of an indenter tip radius is proportional to the CID1 and inversely proportional to the TID. The number of load cycles is inversely proportional to an average of a creep indentation depth (AvCID) and the AvED. The indentation distance increase (IDI) is strongly inversely proportional to the compressive yield stress, and strongly proportional to the viscosity constant and maximum applied load, but has weak relation with the damage parameter, indenter tip radius, and elastic modulus. This computational study advances our understanding of the RPI outputs and provides a starting point for more comprehensive computational studies of the RPI technique.

Keywords: Reference Point Indentation; finite element method; cortical bone; bone strength; bone fracture 


\section{Introduction}

The mechanical properties of bone are generally measured using traditional materials testing approaches such as compression, tension, three or four point bending, and fracture toughness tests. The basic limitations of these methods are that they are ex-vivo and destructive. The Reference Point Indentation (RPI) technique was invented to allow in-vivo measurements of bone material properties, relevant to the risk of bone fracture (Hansma et al., 2006; Diez-Perez et al., 2010). The instrument utilizes a cyclic loading to indent cortical bone multiple times at the same location (Figure 1). The force-versus-displacement response, generated by the RPI technique (Figure 2), allows the calculation of ten RPI parameters. The ID1 and TID are the maximum indentation depths after the first and last cycle, respectively. The IDI is the indentation distance increase observed between the first and last cycles. The CID1 is the creep distance for the first loading cycle while the AvCID is the average of creep distances over all cycles. The US1 is the unloading slope calculated from the first loading cycle and the average of unloading slopes over all cycles is denoted by AvUS. The LS1 is the loading slope for the first loading cycle, AvLS is the average of loading slopes over all cycles and AvED is the energy dissipated over the third to last loading cycle. These RPI parameters are summarized in Table 1 for easy reference. The BioDent $1000^{\mathrm{TM}} \mathrm{RPI}$ instrument (Active Life Scientific, Inc., Santa Barbara, CA) is an experimental device used to perform the RPI tests and utilizes a software which computes the RPI parameters.

The RPI technique has received considerable interest in the bone mechanics community. There have been nearly fifty journal papers published from several different perspectives since its invention in 2006. Some of these studies have focused on the development of the method (Hansma et al., 2006; Hansma et al., 2008), protocols (Setters and Jasiuk, 2014; Coutts et al., 2015; Jenkins et al., 2015), in vivo studies (Diez-Perez et al., 2010; Aref et al., 2013; GüerriFernández et al., 2013; Farr et al., 2014), and ex vivo studies (Randall et al., 2009; Gallant et al., 2013; Rasoulian et al., 2013; Bart et al., 2014; Granke et al., 2014; Hammond et al., 2014; Milovanovic et al., 2014; Beutel and Kennedy, 2015; Coutts et al., 2015; Granke et al., 2015; Hoffseth et al., 2015; Katsamenis et al., 2015). The RPI technique has also been utilized in dental studies (Yassen et al., 2014) and soft tissue research (Hansma et al., 2009; Tang et al., 2010; Tang et al., 2011; Cheng et al., 2014). An overview of the RPI technique is given in a recent review paper by Allen et al. (2015).

Based on these introductory studies on the RPI technique, the IDI has been identified as the most promising output of the RPI method. Diez-Perez et al. (2010) showed that the IDI correlates well with the incidence of fracture and was able to distinguish between normal and osteoporotic patients. Gallant et al. (2013) found that the IDI is inversely correlated with bone toughness obtained from three point bending and axial compression tests. Rasoulian et al. (2013) studied age-related changes in porcine femoral cortical bone and showed that 
the IDI decreases with age in developing bone. In their studies, Granke et al. (2014) demonstrated that the IDI provides the best correlation between yield stress and toughness. However, in general, it is still not well understood how the RPI parameters are related to the specific bone properties. Thus, there is a need for further studies on the interpretation of the RPI parameters before this technique can be more fully utilized in both research and clinical settings.

Computer simulations can provide additional insight into the RPI technique. To date, there has only been one computational study on the RPI method (Hoffseth et al, 2015), which simulated an indentation of the cortical bone using an Osteoprobe, another RPI device designed with clinical setting in mind. This instrument provides only one output, the bone material strength index (normalized indentation distance increase). Other related computational studies include simulations of nanoindentation of bone (Zhang et al., 2008; Mullins et al., 2009; Zhang et al., 2010; Adam and Swain, 2011, among others). A constitutive law that captures the isotropic viscoelastic-plastic response of bone was proposed by Zhang et al. (2008), and it was subsequently generalized to include damage (Zhang et al., 2010).

In this paper we simulate RPI on human cortical bone, mimicking the BioDent tests, using the finite element method (FEM) available in the commercial software Abaqus (V6.14). Our model utilizes the isotropic viscoelastic-plastic constitutive model proposed by Zhang et al. (2010) for human cortical bone. This constitutive law has eight material constants and we vary four of these constants: (Young's modulus, yield stress, and viscous and damage parameters). Additionally, experimental factors (indentation peak load, number of cycles, and indenter tip radius) are varied in the simulations. The force-versus-displacement response is obtained for each case, post-processed to calculate the RPI parameters, and compared with experimental results (Granke et al., 2014). The effects of the input variables on the RPI outputs are reported, and the relationship between the RPI parameters and the mechanical behavior of bone is investigated to gain a better understanding of the RPI technique.

\section{Methods}

\subsection{Material model}

The plastic damage model of Lubliner et al. (1989) is a general model that incorporates damage in materials and a subsequent reduction in elastic modulus under tensile and compressive strains. RPI performed on cortical bone will locally damage the bone sample, mainly due to compressive loading (Setters and Jasiuk, 2014). Therefore, damage due to tensile strains is neglected in this study. 
Assuming continuum damage, where $d$ is the scalar damage variable, the effective stress in the medium is given by equation (1), (Zhang et al., 2010),

$$
\boldsymbol{\sigma}=[1-d] \boldsymbol{E}^{e l}:\left[\boldsymbol{\varepsilon}-\boldsymbol{\varepsilon}^{p l}\right]
$$

where $\boldsymbol{\varepsilon}$ is the total strain, $\boldsymbol{\varepsilon}^{p l}$ is the plastic strain, and $\boldsymbol{E}^{e l}$ is an elasticity tensor. The elasticity tensor is a function of the elastic modulus (Young's modulus) $E$ and Poisson's ratio $v$ for isotropic materials. The true stress can be interpreted as the stress resulting from an undamaged area fraction $(1-d)$ of the medium. The damage variable $d$ assumes a value of between 0 (undamaged) and 1 (fully damaged), depending on the amount of damage induced during loading. The damage parameter is related to the plastic strain using an exponential function:

$$
d=\gamma\left(1-e^{-\beta \kappa_{c}}\right)
$$

where $\gamma$ and $\beta$ are material parameters (see Table 2) and $\kappa_{c}$ is an equivalent compressive plastic strain.

The elasto-plastic boundary is defined by the Drucker-Prager yield function $F$ given as

$$
F=\bar{q}-3 \alpha \bar{p}-Y \leq 0
$$

where $\bar{p}$ is the effective hydrostatic pressure given by

$$
\bar{p}=\frac{1}{3 \bar{\sigma}}: I
$$

and $\bar{q}$ is the effective equivalent von Mises stress given by

$$
\bar{q}=\sqrt{1.5 \bar{\sigma}^{d e v}: \bar{\sigma}^{d e v}}
$$

where $\bar{\sigma}^{\text {dev }}$ is the effective deviatoric stress.

The yield stress is expressed as

$$
Y=[1-\alpha] \bar{\sigma}_{c}-\left[\frac{[1-\alpha] \bar{\sigma}_{c}}{\bar{\sigma}_{t}}-[1+\alpha]\right]\left\langle\bar{\sigma}_{\max }\right\rangle
$$

where $\bar{\sigma}_{c}$ is a uniaxial compressive failure stress, $\bar{\sigma}_{t}$ is a uniaxial tensile failure stress and $\bar{\sigma}_{\text {max }}$ is a maximum eigenvalue. In equation (6), $\langle\ldots\rangle$ are Macaulay brackets, such that under compressive stress, the second term vanishes and only the first term remains.

The coefficient $\alpha$ is given by 


$$
\alpha=\left[\frac{\sigma_{b 0}}{\sigma_{c 0}}-1\right] /\left[2 \frac{\sigma_{b 0}}{\sigma_{c 0}}-1\right]
$$

where $\sigma_{b 0} / \sigma_{c 0}$ is the ratio of an equi-biaxial compressive yield stress to a uniaxial compressive yield stress.

Non-associated plastic flow for bone is defined by a plastic potential $G$ as

$$
G=\bar{q}-\bar{p} \tan \psi
$$

where $\psi$ is a dilation angle, and $\bar{p}$ and $\bar{q}$ are defined in equations (4) and (5).

This model also implements a Duvaut-Lions type viscoelastic-plastic regularization for equivalent plastic strains $\kappa_{c}$ and $\kappa_{t}$ in compression and tension, respectively, and a scalar damage variable $d$,

$$
\begin{aligned}
\dot{d}^{v p} & =\left[d-d_{v p}\right] / \mu \\
\dot{\kappa}_{c}^{v p} & =\left[\kappa_{c}-\kappa_{c}^{v p}\right] / \mu \\
\dot{\kappa}_{t}^{v p} & =\left[\kappa_{t}-\kappa_{t}^{v p}\right] / \mu
\end{aligned}
$$

where $\mu$ is a viscosity parameter.

The parameters (inputs to model human cortical bone) and their default values, used in this study, are listed in Table 2. $E, \sigma_{c 0}, \gamma$ and $\mu$ were varied one at a time during simulations, keeping all other properties constant as shown in Table 3.

\subsection{Finite Element Model}

An axisymmetric finite element (FE) model was built to simulate indentations on human cortical bone, as illustrated in Figure 3. Due to problem nonlinearities, the finite element analysis was not able to numerically converge when using a FE model that includes less than 50,000 four-node bilinear axisymmetric quadrilateral elements (CAX4). Therefore, the FE model mesh density was increased multiple times to achieve a numerically convergent solution. The final FE model, used in this study, contained 52,780 four-node bilinear axisymmetric quadrilateral elements to represent bone tissue (substrate) as a cylinder of radius $2 \mathrm{~mm}$ and height $2 \mathrm{~mm}$. The conical indenter had a $45^{\circ}$ half angle and was modelled using 2,500 four-node bilinear axisymmetric quadrilateral elements. The size of the substrate was chosen to be about 50 times larger than the indentation depths $(\sim 40 \mu \mathrm{m})$. To preserve simulation accuracy, a biased meshing technique was adopted to ensure that a small enough element size was used near the indentation zone $(\sim 2 \mu \mathrm{m})$, while the elastic properties of steel were used 
to simulate the indenter tip. Human bone material parameters, defined in the isotropic viscoelastic-plastic constitutive model of Zhang et al. (2010), were used in the FE simulations.

\subsection{Boundary conditions}

The bottom edge nodes of the substrate representing a human cortical bone were fully restrained while the vertical edge nodes of the sample were kept free. Interactions between the cortical bone and the indenter tip in the FE model were represented using surface-to-surface and finite sliding algorithms (Abaqus V6.14 theory guide). The friction coefficient between the indenter tip and the cortical bone sample is not well defined in the literature and is very sensitive to the indenter tip and bone sample surface finish. In this study the friction coefficient was assumed to be 0.1 . The indentation force and number of cycles were varied as shown in Table 3.

\subsection{Analysis and Post-processing}

Due to the large deformations and contact between the indenter and the bone sample, a nonlinear FEA was performed, where geometric and contact nonlinearities were included.

$\mathrm{RPI}$ is a dynamic problem and therefore the analysis was initially performed using dynamic solvers. Abaqus offers two dynamic solvers: implicit and explicit. Using the Abaqus explicit solver required a long analysis time for each iteration (over 50 hours when using 64CPUs $2.9 \mathrm{GHz}$ each). Also the dynamic implicit solver demanded a longer analysis time than using the quasi-static implicit solver. The RPI dynamic analysis showed that problem kinetic energy is lower than $1 \%$ compared to its internal energy (recoverable strain energy + plastic dissipation energy); this result was expected because the initial distance between the indenter tip and the cortical bone sample is very small, mimicking the actual $\mathrm{RPI}$ test setup. Therefore, the analysis was performed using a quasi-static implicit solver, based on our earlier study (Idkaidek and Jasiuk, 2015). The RPI simulations using the quasi-static analysis generated very similar results to those obtained using the dynamic analysis but the problem analysis time was reduced by approximately $30 \%$ compared to the implicit dynamic analysis and over $60 \%$ compared to the explicit dynamic analysis. Thus, the RPI simulations were conducted using the quasi-static model within the Abaqus (version 6.14) software package.

The RPI parameters were computed from the force-displacement data. The BioDent instrument software calculates the unloading slopes by fitting a line to data points between $5 \%$ and $40 \%$ of the unloading time interval. Loading slopes are calculated by fitting a line to the data points obtained in the last $40 \%$ of the loading time interval. Loading and unloading slopes calculated from the 
simulation results followed the same method as in the BioDent instrument software.

\section{Results and Discussion}

\subsection{Computational Results}

In this paper we investigated computationally the effect of various material and testing variables on the RPI parameters. The RPI technique has been used to measure bone material properties in-vivo to predict the risk of bone fracture (Hansma et al., 2006; Diez-Perez et al., 2010). However, the relationship between the RPI measured parameters and bone properties is still not well understood. To address this issue, we simulated the RPI on human cortical bone, mimicking BioDent tests. Cortical bone material inputs $\left(E, \sigma_{c 0}, \gamma\right.$ and $\left.\mu\right)$ and testing characteristics (cyclic peak force, number of cycles, and indenter tip radius) were varied one at a time during simulations as summarized in Table 3, while all other inputs remained constant as shown in Tables 2 and 3 in a bold font. Thus, we studied how various forms of the stress-strain curve and testing variables influence the RPI parameters. Note that, for simplicity, we did not model bone fracture due to indentation.

In order to gain an insight into local fields due to the RPI indentations, Figure 4 shows the RPI simulation results obtained using the default inputs (as given in Tables 2 and 3 in a bold font). Figure 4-a illustrates the von Mises stress contours at the peak load of the 5th loading cycle due to RPI while Figure 4-b shows the equivalent plastic strain contours at the end of the 5th loading cycle. It is evident from the contour plots that damage is contained within a small zone close to the indenter $(\sim 50 \mu \mathrm{m}-150 \mu \mathrm{m})$.

The RPI finite element simulations show that an increase in the elastic modulus leads to decreases in the ID1 and TID outputs and to increases in the US1, LS1, AvUS, and AvLS outputs. This behavior is expected because an increase in Young's modulus will increase the resistance of bone to elastic deformations due to applied forces, which results in an increase in the slope of the force-deformation curves. Interestingly, the IDI does not follow this trend and shows little dependence on Young's modulus. Varying elastic modulus also has a limited effect on the CID1, AvCID and AvED outputs, which is expected since these are associated with time-dependent material behavior (creep and energy dissipation). These results are summarized in Table 4.

Secondly, an increase in the compressive yield stress decreases the ID1, CID1, TID, AvCID, and AvED parameters and increases the LS1, but has limited effect on the US1, AvUS, and AvLS parameters. We also found that increasing the compressive yield stress leads to a decrease in the IDI, indicating that increasing the compressive yield stress reduces the change in bone deformation 
during RPI loading. This behavior is consistent with expectations. The effects of varying the compressive yield stress on the RPI parameters are summarized in Table 5. Figure 5 shows the force vs displacement responses where the material becomes more compliant and indentation depths are higher as the elastic modulus (Fig. 5a) and compressive yield stress (Fig. 5b) are decreased.

Third, if the damage constant is increased there is an increase in the ID1, CID1, and TID parameters, resulting in deeper indents, again as expected. Also, increasing the damage constant causes decreases in the US1, LS1, AvUS, AvLS, and AvED parameters. This implies that damage is initiated early on due to the sharp tip of the indenter. Interestingly, the effect of increasing the damage constant shows no clear trends on the IDI and AvCID. For example, increasing the damage parameter by $60 \%$ and $80 \%$ decreased the IDI by only $22 \%$ and $11 \%$, respectively. The effects of variation of the damage constant on RPI parameters and the force vs. deformation are shown in Table 6 and Figure 6-a, respectively.

Next, an increase in the viscosity constant leads to increases in the CID1, AvCID and AvED as expected since these represent time-dependent characteristics of the force-deformation curve. Increasing the viscosity constant also leads to an increase in the IDI. Furthermore, an increase in the viscosity constant leads to decreases in the ID1 and TID; it is interesting that these have an opposite trend to the IDI results. The slopes LS1, AvUS, and AvLS show no dependence on the viscosity constant while US1 shows a slight increase; Table 7 and Figure 6-b show the effect of variation of the viscosity constant.

The RPI maximum applied load is found to be proportional to all RPI parameters including the IDI, where higher peak loads result in larger indentation distances. Table 8 and Figure 7-a illustrate the effect of different cyclic peak loads on RPI parameters and the force versus displacement response.

Furthermore, we find that the indenter radius is proportional to CID1 and AvCID values, where increasing the indenter tip radius from $15 \mu \mathrm{m}$ to $30 \mu \mathrm{m}$ increased the CID1 and AvCID values by $7 \%$ and $5 \%$, respectively. When the indenter tip radius is increased there is a decrease in the ID1, TID, and AvED, but there is a limited effect on the LS1, US1, AvLS, and AvUS. Also increasing indenter tip radius by $50 \%$ has a limited effect on the IDI. Table 9 and Figure 7-b show the effect of varying the indenter tip radius.

A strong linear correlation between the US1 and the AvUS versus elastic modulus is observed, which shows that the US1 can be used to determine the elastic modulus. Decreasing the elastic modulus by $8.6 \%$ and $13.98 \%$ decreased the US1 by $4.92 \%$ and $8.20 \%$, respectively. Also, the US1 is relatively insensitive to changes in the compressive yield stress, where a $50 \%$ change in the compressive yield stress resulted in almost no change in the US1. This is expected because the material response at the beginning of unloading is mainly 
elastic. A linear relationship is also observed between the elastic modulus versus the LS1 and the AvLS, where a decrease in the elastic modulus of $8.6 \%$ and $24.73 \%$ decreased the AvLS by $4 \%$ and $12 \%$, respectively. Unloading slopes (both US1 and AvUS) were also found to be inversely proportional to the change in the damage parameter and proportional to the peak force, as observed in Tables 6 and 8.

Decreases in the elastic modulus, compressive yield stress, and viscous parameters and an increase in the damage parameter correspond with the increase in the indentation distances ID1 and TID. This is an important finding, which correlates with earlier reports that showed that indentation distances capture the inelastic behavior of bone.

As mentioned in the Introduction, the IDI has been identified as the most promising RPI parameter to assess the susceptibility of bone to fracture. Our computations show that the IDI is not a strong function of the elastic modulus and the indenter tip radius, as illustrated in Tables 4 and 9. The fact that the IDI is insensitive to the indenter tip radius is an important observation since it is known that the indenter tip blunts with additional measurements. On the other hand, the IDI varies with the change in the compressive yield stress, viscosity constant, and indentation load value. The IDI is found to be proportional to the viscosity constant and indentation load value and inversely proportional to the compressive yield stress, as shown in Tables 5, 7, and 8, and also in Figure 8. These trends are expected. However, it is interesting to note that the IDI does not have a consistent trend with the damage constant for the choice of parameters that were used in our simulations. A more complete parametric investigation could provide further insights.

Creep distances (CID1 and AvCID) are found to be inversely proportional to the compressive yield stress and only the AvCID is inversely proportional to the cyclic peak force. On the other hand, creep distances are proportional to the viscosity constant and indenter tip radius as shown in Tables 5, 7, 9, and 10.

The RPI simulations were performed using a $10 \mathrm{~N}$ cyclic load and the defaults for all other inputs (as shown in Tables 2 and 3 in bold font) were used to investigate the effect of the number of cycles on the RPI parameters. Results for the variation in the number of cycles are presented in Table 10, where the ID1, US1, LS1, and CID1 do not change due to their dependency on the first cycle only. Also the changes in TID and AvUS are small, which is expected due to the energy dissipation decrease as the number of load cycles increases. Also the AvCID and AvED were found to be inversely proportional to the number of load cycles.

Existing experimental studies have sought correlations between the RPI parameters and mechanical properties such as elastic modulus (stiffness), yield strain or stress, toughness, fracture toughness, or ultimate 
strength. Researchers found a positive relationship between the stiffness and AvLS and AvUS (Granke et al. 2014) and a negative relationship between postyield strain and IDI (Hansma et al 2008; Granke et al. 2014), which agree with the findings of our study. They also found negative relations between the IDI and toughness (Hansma et al. 2008; Granke et al. 2014), the IDI and crack growth toughness (Diez-Perez et al. 2010; Katsamenis et al. 2015) and the IDI and ultimate strength (Granke et al. 2014; Abraham et al. 2015), among others. However, since our computational model does not include bone tissue fracture, we could not study correlations of the RPI parameters with the bone strength, toughness, and fracture toughness.

Bone exhibits complex failure phenomena due to its complex composite, inhomogeneous and hierarchical structure. Various intrinsic (plastic deformation) and extrinsic (crack-tip shielding) toughening mechanisms are present in cortical bone during deformation (Launey et al, 2010). Intrinsic mechanisms include molecular uncoiling of collagen and intermolecular sliding, microcracking, and fibrillar sliding at the mineralized collagen fibril level. At higher structural scales, extrinsic mechanisms, which include crack deflection and twisting, collagen fibril bridging and constrained microcracking, are more pronounced. At a yet higher scale, osteons, cement lines, and porosities such as Haversian canals contribute to the bone's resistance to fracture. Thus, there are complex elastic, viscoelastic and plastic deformations that take place in bone at different structural scales and their collective interplay contributes to the bone quality (Launey et al., 2010; Sabet et al., 2016). We employed a constitutive law at the cortical bone tissue level, which includes elastic, viscoelastic and plastic components to account for, in an average sense, these complex deformations that occur at different structural levels. A more detailed multiscale model of the RPI indentation, including modeling of the bone fracture, could provide further insights into the contributions of these multiscale phenomena on the RPI outputs.

This study has several limitations. The constitutive law used (Zhang et al., 2010) implements a continuum damage model that does not include fracture. We did not model cracks explicitly and did not account for fracture of the cortical bone tissue due to indentations. On the contrary, scanning electron microscopy images (Setters and Jasiuk, 2014) of bone subjected to RPI showed cracks. Moreover, this study represents bone as a homogeneous and isotropic material, whereas it is known that bone has a complex spatially heterogeneous microstructure and exhibits anisotropy. We used both assumptions for computational simplicity. Future studies can provide more accurate RPI simulation results after releasing such assumptions. Thus, the observations reported in this paper are within the constraints of the model used in simulations, which assumes isotropy and includes plasticity and damage but no fracture. Also, we did not employ a multiscale model that would account for a hierarchical structure of bone. Again, this was done for simplicity. Another limitation is neglecting damage due to tensile strains; however, RPI on bone is expected to induce damage that is mainly due to compressive stresses, as indicated in Figure 9. 


\subsection{Comparison with experiments}

Granke et al. (2014) performed RPI experiments using the BioDent, applying a cyclic load of $10 \mathrm{~N}$ in two orthogonal directions (longitudinal and transverse) on the femoral mid-shafts from 26 human donors 25-101 years old. In their study the experimentally obtained RPI parameters (CID1, LS1, US1, AvCID, AvLS, and AvUS) were reported for transverse and longitudinal indentations. We use these results to validate our computational results. In this paper the RPI simulations were performed using the FE model described in Figure 3 , the boundary conditions described in Section 2.2 and human bone isotropic viscoelastic-plastic constitutive model proposed by Zhang et al. (2010). We used the material properties described in Tables 2 and 3 in bold font, and 10 load cycles as described in Figure 1 with a $10 \mathrm{~N}$ peak load value. In order to investigate the effect of the indenter tip radius on the RPI simulation results, two RPI simulation iterations were performed using $30 \mu \mathrm{m}$ and $15 \mu \mathrm{m}$ indenter tip radii.

Table 11 compares the computational results with the experimental results of Granke et al. (2014). The RPI simulation results agree to within $0 \%$ to $42 \%$ with the experimental results. Furthermore, the simulation results agree (0\% to $25 \%)$ with the test results if LS1 is disregarded. The simulation results are about $40 \%$ higher for the LS1 values compared to the experimental results. This is probably due to the presence of pores in the indentation area as described in the experimental study (Granke et al., 2014). It is important to note that we are assuming isotropic material properties while the test results were reported in two orthogonal directions (longitudinal and transverse). Our assumption is expected to have a negative effect on the comparison with test results. On the other hand, all RPI parameters computed from the simulations are within the reported experimental values for both transverse and longitudinal indentations.

\section{Conclusions}

The finite element method within Abaqus software V6.14 was used to simulate RPI on human cortical bone and provided new data on how the RPI parameters are related to the material constants used to represent human bone properties (Zhang et al., 2010). Simulation results are in good agreement with the experimental study of Granke et al. (2014).

The simulation results indicate that RPI parameters are sensitive to the material properties of human cortical bone. The unloading slopes were found to be good indicators of the elastic modulus. ID1 and TID parameters have the same strong relationship to the material constants, where both are inversely proportional to the elastic modulus, compressive yield stress, viscous constant, and indenter tip radius. On the other hand, ID1 and TID are proportional to the 
damage parameter and the maximum applied load. Also the IDI is found to have a limited relation to the damage parameter, indenter tip radius, and elastic modulus. However, IDI was found to be strongly inversely proportional to the compressive yield stress, and strongly proportional to the viscosity constant and maximum applied load.

These simulations provide new insights into the RPI technique, which can generate data on bone material properties. Further insights can be obtained by conducting more accurate simulations and doing further experiments using the $\mathrm{RPI}$ technique. Such studies are needed before the RPI method becomes more widely accepted and utilized.

\section{Acknowledgments}

The authors gratefully acknowledge support from the National Science Foundation (NSF), the CMMI Program Grant 09-27909 and the DMR Program Grant 15-07169. The findings, conclusions and recommendations expressed in this manuscript are those of the authors and do not necessarily reflect the views of the NSF.

\section{References}

ABAQUS V6.14 theory guide. http://abaqus.software.polimi.it/v6.14/books/stm/default.htm

Abraham AC, A. Agarwalla, A. Yadavalli, C. McAndrew, J.Y. Liu and S.Y. Tang (2015). "Multiscale Predictors of Femoral Neck In Situ Strength in Aging Women: Contributions of BMD, Cortical Porosity, Reference Point Indentation, and Nonenzymatic Glycation." Journal of Bone and Mineral Research 30: 2207-2214.

Adam, C.J. and M.V. Swain (2011). "The effect of friction on indenter force and pile-up in numerical simulations of bone nanoindentation." Journal of the Mechanical Behavior of Biomedical Materials 4(7): 1554-1558.

Allen, M.R., E.M.B. McNerny, J.M. Organ and J.M. Wallace (2015). "True Gold or Pyrite: A review of reference point indentation for assessing bone mechanical properties in vivo." Journal of Bone and Mineral Research 30(9): 1539-1550.

Aref, M., M.A. Gallant, J.M. Organ, J.M. Wallace, C.L. Newman, D.B. Burr, D.M. Brown and M.R. Allen (2013). "In vivo reference point indentation reveals positive effects of raloxifene on mechanical properties following 6 months of treatment in skeletally mature beagle dogs." Bone 56(2): 449-453. 
Bart, Z.R., M.A. Hammond and J.M. Wallace (2014). "Multi-scale analysis of bone chemistry, morphology and mechanics in the OIM model of osteogenesis imperfecta." Connective Tissue Research 55: 4-8.

Beutel, B.G. and O.D. Kennedy (2015). "Characterization of damage mechanisms associated with reference point indentation in human bone." Bone 75: $1-7$.

Cheng, K.K., S.H. Berven, S.S. Hu and J.C. Lotz (2014). "Intervertebral discs from spinal nondeformity and deformity patients have different mechanical and matrix properties." The Spine Journal 14(3): 522-530.

Coutts, L.V., T. Jenkins, T. Li, D.G. Dunlop, R.O.C. Oreffo, C. Cooper, N.C. Harvey, P.J. Thurner and O.S. Grp (2015). "Variability in reference point rnicroindentation and recommendations for testing cortical bone: Location, thickness and orientation heterogeneity." Journal of the Mechanical Behavior of Biomedical Materials 46: 292-304.

Diez-Perez, A., R. Guerri, X. Nogues, E. Caceres, M. Jesus Pena, L. Mellibovsky, C. Randall, D. Bridges, J.C. Weaver, A. Proctor, D. Brimer, K.J. Koester, R.O. Ritchie and P.K. Hansma (2010). "Microindentation for in vivo measurement of bone tissue mechanical properties in humans." Journal of Bone and Mineral Research 25(8): 1877-1885.

Farr, J.N., M.T. Drake, S. Amin, L.J. Melton, III, L.K. McCready and S. Khosla (2014). "In vivo assessment of bone quality in postmenopausal women with type 2 diabetes." Journal of Bone and Mineral Research 29(4): 787-795.

Gallant, M.A., D.M. Brown, J.M. Organ, M.R. Allen and D.B. Burr (2013). "Reference-point indentation correlates with bone toughness assessed using whole-bone traditional mechanical testing." Bone 53(1): 301-305.

Granke, M., A. Coulmier, S. Uppuganti, J.A. Gaddy, M.D. Does and J.S. Nyman (2014). "Insights into reference point indentation involving human cortical bone: Sensitivity to tissue anisotropy and mechanical behavior." Journal of the Mechanical Behavior of Biomedical Materials 37: 174-185.

Granke, M., A.J. Makowski, S. Uppuganti, M.D. Does and J.S. Nyman (2015). "Identifying novel clinical surrogates to assess human bone fracture toughness." Journal of Bone and Mineral Research 30(7): 1290-1300.

Güerri-Fernández, R.C., X. Nogués, J.M. Quesada Gómez, E. Torres del Pliego, L. Puig, N. García-Giralt, G. Yoskovitz, L. Mellibovsky, P.K. Hansma and A. DíezPérez (2013). "Microindentation for in vivo measurement of bone tissue material 
properties in atypical femoral fracture patients and controls." Journal of Bone and Mineral Research 28(1): 162-168.

Hammond, M.A., M.A. Gallant, D.B. Burr and J.M. Wallace (2014). "Nanoscale changes in collagen are reflected in physical and mechanical properties of bone at the microscale in diabetic rats." Bone 60: 26-32.

Hansma, P., P. Turner, B. Drake, E. Yurtsev, A. Proctor, P. Mathews, J. Lelujian, C. Randall, J. Adams, R. Jungmann, F. Garza-de-Leon, G. Fantner, H. Mkrtchyan, M. Pontin, A. Weaver, M.B. Brown, N. Sahar, R. Rossello and D. Kohn (2008). "The bone diagnostic instrument II: Indentation distance increase." Review of Scientific Instruments 79(6).

Hansma, P., H. Yu, D. Schultz, A. Rodriguez, E.A. Yurtsev, J. Orr, S. Tang, J. Miller, J. Wallace, F. Zok, C. Li, R. Souza, A. Proctor, D. Brimer, X. NoguesSolan, L. Mellbovsky, M.J. Peña, O. Diez-Ferrer, P. Mathews, C. Randall, A. Kuo, C. Chen, M. Peters, D. Kohn, J. Buckley, X. Li, L. Pruitt, A. Diez-Perez, T. Alliston, V. Weaver and J. Lotz (2009). "The tissue diagnostic instrument." Review of Scientific Instruments 80(5): 054303.

Hansma, P.K., P.J. Turner and G.E. Fantner (2006). "Bone diagnostic instrument." Review of Scientific Instruments 77(7).

Hoffseth, K., C. Randall, P. Hansma and H.T.Y. Yang (2015). "Study of indentation of a sample equine bone using finite element simulation and single cycle reference point indentation." Journal of the Mechanical Behavior of Biomedical Materials 42: 282-291.

Idkaidek, A. and I. Jasiuk (2015). "Toward high-speed 3D nonlinear soft tissue deformation simulations using Abaqus software." Journal of Robotic Surgery 9(4):299-310.

Jenkins, T., L.V. Coutts, D.G. Dunlop, R.O.C. Oreffo, C. Cooper, N.C. Harvey, P.J. Thurner and O.S. Grp (2015). "Variability in reference point microindentation and recommendations for testing cortical bone: Maximum load, sample orientation, mode of use, sample preparation and measurement spacing." Journal of the Mechanical Behavior of Biomedical Materials 42: 311-324.

Katsamenis, O.L., T. Jenkins and P.J. Thurner (2015). "Toughness and damage susceptibility in human cortical bone is proportional to mechanical inhomogeneity at the osteonal-level." Bone 76: 158-168.

Launey, M.E., M.J. Buehler, and R.O. Ritchie (2010), "On the mechanistic origins of toughness in bone." Annual Review of Materials Research 40: 25-53. 
Lubliner, J., J. Oliver, S. Oller and E. Oñate (1989). "A plastic-damage model for concrete." International Journal of Solids and Structures 25(3): 299-326.

Milovanovic, P., Z. Rakocevic, D. Djonic, V. Zivkovic, M. Hahn, S. Nikolic, M. Amling, B. Busse and M. Djuric (2014). "Nano-structural, compositional and micro-architectural signs of cortical bone fragility at the superolateral femoral neck in elderly hip fracture patients vs. healthy aged controls." Experimental Gerontology 55: 19-28.

Mullins, L.P., M.S. Bruzzi and P.E. McHugh (2009). "Calibration of a constitutive model for the post-yield behaviour of cortical bone." Journal of the Mechanical Behavior of Biomedical Materials 2(5): 460-470.

Randall, C., P. Mathews, E. Yurtsev, N. Sahar, D. Kohn and P. Hansma (2009). "The bone diagnostic instrument III: Testing mouse femora." Review of Scientific Instruments 80(6).

Rasoulian, R., A.R. Najafi, M. Chittenden and I. Jasiuk (2013). "Reference point indentation study of age-related changes in porcine femoral cortical bone." Journal of Biomechanics 46(10): 1689-1696.

Sabet, F., A. Najafi Raeisi, E. Hamed, and I. Jasiuk (2016), "Modelling of bone fracture and strength at different length scales - A Review," Interface Focus 6 (1), 20150055.

Setters, A. and I. Jasiuk (2014). "Towards a standardized reference point indentation testing procedure." Journal of the Mechanical Behavior of Biomedical Materials 34: 57-65.

Tang, S.Y., P. Mathews, C. Randall, E. Yurtsev, K. Fields, A. Wong, A.C. Kuo, T. Alliston and P. Hansma (2010). "In situ materials characterization using the tissue diagnostic instrument." Polymer Testing 29(2): 159-163.

Tang, S.Y., R.B. Souza, M. Ries, P.K. Hansma, T. Alliston and X. Li (2011). "Local tissue properties of human osteoarthritic cartilage correlate with magnetic resonance T1rho relaxation times." Journal of Orthopaedic Research 29(9): 1312-1319.

Yassen, G.H., S.S. Al-Angari and J.A. Platt (2014). "The use of traditional and novel techniques to determine the hardness and indentation properties of immature radicular dentin treated with antibiotic medicaments followed by ethylenediaminetetraacetic acid." European Journal of Dentistry 8(4): 521-527.

Zhang, J., M.M. Michalenko, E. Kuhl and T.C. Ovaert (2010). "Characterization of indentation response and stiffness reduction of bone using a continuum damage 
model." Journal of the Mechanical Behavior of Biomedical Materials 3(2): 189202.

Zhang, J., G.L. Niebur and T.C. Ovaert (2008). "Mechanical property determination of bone through nano- and micro-indentation testing and finite element simulation." Journal of Biomechanics 41(2): 267-275. 


\section{Symbols:}

\begin{tabular}{|c|l|}
\hline & RPI parameter increase \\
\hline$\downarrow$ & RPI parameter decrease \\
\hline$\sim$ & RPI parameter slightly increase \\
\hline$\sim$ & RPI parameter slightly decrease \\
\hline-- & RPI parameter did not change \\
\hline$\sim$ & No trend in RPI parameter behavior \\
\hline
\end{tabular}

\section{List of Tables:}

Table 1. Description of RPI outputs.

Table 2. Base material properties of human cortical bone, load values, and number of loading cycles.

Table 3. Additional material properties, load values, and number of loading cycles.

Table 4. Computationally obtained RPI parameters for different values of Young's modulus.

Table 5. Computed RPI parameters as a function of compressive yield stress.

Table 6. Computed RPI parameters as a function of material damage constant (Y).

Table 7. Computed RPI parameters as a function of viscosity constant.

Table 8. Computed RPI parameters as a function of maximum applied cyclic force.

Table 9. Computed RPI parameters as a function of indenter tip radius.

Table 10. Computed RPI parameters as a function of number of cycles.

Table 11. Comparison between RPI experimental results (Granke et al., 2014) and computed $\mathrm{RPI}$ results ( $r$ : indenter tip radius).

\section{List of Figures:}

Figure 1. RPI cyclic loading. a) Typical 5 cycles of RPI loading. b) Loading function.

Figure 2. Typical force vs displacement curves for RPI first and last loading cycles with parameters.

Figure 3. Axisymmetric (FE) model of RPI.

Figure 4. RPI simulation results at the end of the $5^{\text {th }}$ loading cycle using material properties as given in Tables 2 and 3 in bold font. a) Von Mises stress contours.

b) Equivalent plastic strain contours.

Figure 5. RPI simulations results: a) Young's modulus effect. b) Compressive yield stress effect.

Figure 6. RPI simulations results: a) Damage constant effect. b) Viscosity constant effect.

Figure 7. RPI simulations results: a) Applied peak cyclic force effect. b) Indenter tip radius effect. 
Figure 8. IDI relation to compressive yield stress, viscosity constant, and indentation load value.

Figure 9. Stress contours at the end of $1^{\text {st }}$ cycle loading using material properties as given in Tables 2 and 3 in bold font: a) $\sigma_{11}$ and b) $\sigma_{22}$. The contours shown in the image are mainly in a dark color (black), which represents compressive stresses. Tensile stresses are represented mainly in green. The tensile stresses are many orders smaller compared to the compressive stresses, as shown in the figure. 


\section{Tables:}

Table 1. Description of RPI outputs.

\begin{tabular}{|c|l|}
\hline RPI Output & Description \\
\hline ID1 & Maximum first cycle indentation depth \\
\hline LS1 & First loading cycle force vs displacement loading slope \\
\hline US1 & First loading cycle force vs displacement unloading slope \\
\hline CID1 & Creep distance for the first loading cycle \\
\hline TID & Total test probe penetration depth \\
\hline IDI & Indentation distance increase from first cycle to last cycle \\
\hline AvCID & Average of creep indentation depth over all cycles \\
\hline AvUS & Average force vs displacement unloading slope over all cycles \\
\hline AvLS & Average force vs displacement loading slope over all cycles \\
\hline AvED & Average of energy dissipated over $3^{\text {rd }}$ to last test cycles \\
\hline
\end{tabular}

Table 2. Base material properties of human cortical bone, load values, and number of loading cycles.

\begin{tabular}{|c|c|l|}
\hline Parameter & Value & Description \\
\hline $\boldsymbol{\psi}$ & $22.5^{\circ}$ & Dilation angle \\
\hline$\frac{\sigma_{\boldsymbol{b} \mathbf{0}}}{\boldsymbol{\sigma}_{\boldsymbol{c} \mathbf{0}}}$ & 1.125 & $\begin{array}{l}\text { Ratio of equibiaxial compressive yield } \\
\text { stress to uniaxial compressive yield } \\
\text { stress. }\end{array}$ \\
\hline $\boldsymbol{\sigma}_{\boldsymbol{c} \mathbf{0}}$ & $100 \mathrm{MPa}$ & Uniaxial Compressive Yield Stress \\
\hline $\boldsymbol{E}$ & $18.6 \mathrm{GPa}$ & Elastic Modulus \\
\hline $\boldsymbol{v}$ & 0.3 & Poisson's Ratio \\
\hline $\boldsymbol{\gamma}$ & 0.9 & Constant for calculation of $d$ using Eq 3 \\
\hline $\boldsymbol{\beta}$ & 19 & Constant for calculation of $d$ using Eq 3 \\
\hline $\boldsymbol{\mu}$ & 0.04 & Viscosity constant - seconds \\
\hline $\boldsymbol{F}$ & 8 & Force - Newton \\
\hline Cycles & 5 & Number of cycles \\
\hline
\end{tabular}


Table 3. Additional material properties, load values, and number of loading cycles.

\begin{tabular}{|c|c|}
\hline Constants & Values \\
\hline$E$ & 14GPa, 15GPa, 16GPa, 17GPa, 18.6GPa, \\
\hline$\sigma_{c 0}$ & 50MPa, 75MPa, 100MPa,150MPa, 200MPa \\
\hline$\gamma$ & $0.5,0.8,0.9$ \\
\hline $\mathbf{F}$ & $6 \mathrm{~N}, \mathbf{8 N}, 10 \mathrm{~N}$ (N: Newton) \\
\hline $\boldsymbol{\mu}$ & $0.02 s, 0.04 s, 0.08 s$ (s: seconds) \\
\hline Cycles & $2,5,7,10$ \\
\hline Indenter tip radios & $15 \mu \mathrm{m}, \mathbf{3 0} \boldsymbol{\mu m}$ ( $\boldsymbol{\mu m}:$ micro meter $)$ \\
\hline
\end{tabular}

Table 4. Computationally obtained RPI parameters for different values of Young's modulus.

\begin{tabular}{|c|c|c|c|c|c|c|}
\hline \multirow{2}{*}{$\begin{array}{c}\text { RPI } \\
\text { parameters }\end{array}$} & \multicolumn{6}{|c|}{ Young modulus } \\
\hline & $\mathrm{E}=14 \mathrm{GPa}$ & $\mathrm{E}=15 \mathrm{GPa}$ & $\mathrm{E}=16 \mathrm{GPa}$ & $\mathrm{E}=17 \mathrm{GPa}$ & $\mathrm{E}=18.6 \mathrm{GPa}$ & Trend \\
\hline ID1 ( $\mu \mathrm{m})$ & 38.14 & 36.66 & 35.33 & 34.19 & 32.46 & \\
\hline 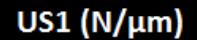 & 0.52 & 0.55 & 0.56 & 0.58 & 0.61 & 1 \\
\hline LS1 (N/ $\mu \mathrm{m})$ & 0.24 & 0.24 & 0.25 & 0.25 & 0.26 & 1 \\
\hline CID1 ( $\mu \mathrm{m})$ & 5.09 & 4.85 & 4.91 & 5.08 & 5.02 & $\sim$ \\
\hline TID $(\mu \mathrm{m})$ & 43.88 & 42.48 & 41.21 & 39.97 & 38.45 & 1 \\
\hline IDI ( $\mu \mathrm{m})$ & 5.54 & 5.48 & 5.62 & 5.62 & 5.71 & $\sim$ \\
\hline AvCID $(\mu \mathrm{m})$ & 1.09 & 1.02 & 1.06 & 1.09 & 1.08 & $\sim$ \\
\hline AvUS (N/ $\mu \mathrm{m})$ & 0.50 & 0.52 & 0.54 & 0.56 & 0.59 & 1 \\
\hline AvLS (N/ $\mu \mathrm{m})$ & 0.44 & 0.45 & 0.46 & 0.48 & 0.50 & 1 \\
\hline AvED (N $\mu m)$ & 2.14 & 1.96 & 2.16 & 2.13 & 2.16 & $\sim$ \\
\hline
\end{tabular}

Table 5. Computed RPI parameters as a function of compressive yield stress.

\begin{tabular}{|c|c|c|c|c|c|c|}
\hline \multirow[b]{2}{*}{ RPI parameters } & \multicolumn{6}{|c|}{ Compressive Yield Stress } \\
\hline & $\sigma_{C 0}=50 M P a$ & $\sigma_{C 0}=75 \mathrm{MPa}$ & $\sigma_{C 0}=100 M P a$ & $\sigma_{C 0}=150$ & $0=200$ & Trend \\
\hline ID1 ( $\mu \mathrm{m})$ & 35.24 & 33.66 & 32.46 & 30.96 & 29.91 & $\downarrow$ \\
\hline US1 $(\mathrm{N} / \mu \mathrm{m})$ & 0.67 & 0.62 & 0.61 & 0.61 & 0.61 & $\sim$ \\
\hline 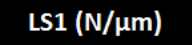 & 0.24 & 0.25 & 0.26 & 0.28 & 0.29 & $\boldsymbol{1}$ \\
\hline CID1 ( $\mu \mathrm{m})$ & 7.57 & 6.00 & 5.02 & 4.04 & 3.48 & $\downarrow$ \\
\hline TID $(\mu \mathrm{m})$ & 47.43 & 41.29 & 38.45 & 35.78 & 34.19 & 7 \\
\hline IDI ( $(\mu \mathrm{m})$ & 12.09 & 7.45 & 5.71 & 4.54 & 4.06 & 7 \\
\hline AvCID $(\mu \mathrm{m})$ & 2.04 & 1.40 & 1.08 & 0.86 & 0.73 & 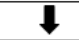 \\
\hline AvUS $(N / \mu \mathrm{m})$ & 0.65 & 0.60 & 0.59 & 0.57 & 0.56 & 7 \\
\hline AvLS $(\mathrm{N} / \mu \mathrm{m})$ & 0.50 & 0.50 & 0.50 & 0.49 & 0.50 & -- \\
\hline AvED (N $\mathrm{mm})$ & 7.58 & 3.31 & 2.16 & 1.59 & 1.23 & $\nabla$ \\
\hline
\end{tabular}


Table 6. Computed RPI parameters as a function of material damage constant $(\gamma)$.

\begin{tabular}{|c|c|c|c|c|}
\hline \multirow{2}{*}{$\begin{array}{c}\text { RPI } \\
\text { parameters }\end{array}$} & \multicolumn{4}{|c|}{ Constant for calculation of $d$ using Eq 3} \\
\hline & $\gamma=0.5$ & $\gamma=0.8$ & $\gamma=0.9$ & Trend \\
\hline ID1 ( $\mu \mathrm{m})$ & 24.92 & 29.59 & 32.46 & $\boldsymbol{1}$ \\
\hline US1 (N/ $\mu \mathrm{m})$ & 0.88 & 0.66 & 0.61 & $\downarrow$ \\
\hline LS1 (N/ $/ \mu \mathrm{m})$ & 0.36 & 0.29 & 0.26 & $\downarrow$ \\
\hline CID1 ( $\mu \mathrm{m})$ & 4.10 & 4.18 & 5.02 & $\boldsymbol{1}$ \\
\hline TID $(\mu \mathrm{m})$ & 31.48 & 34.72 & 38.45 & $\boldsymbol{1}$ \\
\hline IDI ( $\mu \mathrm{m})$ & 6.42 & 5.01 & 5.71 & $\sim$ \\
\hline $\operatorname{AvCID}(\mu \mathrm{m})$ & 1.19 & 1.02 & 1.08 & $\sim$ \\
\hline AvUS (N/ $/ \mathrm{m})$ & 1.01 & 0.70 & 0.59 & 1 \\
\hline AvLS $(\mathrm{N} / \mu \mathrm{m})$ & 0.80 & 0.59 & 0.50 & 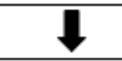 \\
\hline AvED $(\mathrm{N} \mu \mathrm{m})$ & 5.25 & 3.26 & 2.16 & $\downarrow$ \\
\hline
\end{tabular}

Table 7. Computed RPI parameters as a function of viscosity constant.

\begin{tabular}{|c|c|c|c|c|}
\hline \multirow[b]{2}{*}{ RPI parameters } & \multicolumn{4}{|c|}{ Viscosity constant } \\
\hline & $\mu=0.02$ & $\mu=0.04$ & $\mu=\mathbf{0 . 0 8}$ & Trend \\
\hline ID1 ( $\mu \mathrm{m})$ & 34.77 & 32.46 & 29.22 & $\downarrow$ \\
\hline US1 (N/ $\mu \mathrm{m})$ & 0.60 & 0.61 & 0.64 & $\boldsymbol{1}$ \\
\hline LS1 $(\mathrm{N} / \mu \mathrm{m})$ & 0.26 & 0.26 & 0.27 & -- \\
\hline CID1 $(\mu \mathrm{m})$ & 3.34 & 5.02 & 6.55 & $\boldsymbol{1}$ \\
\hline TID $(\mu \mathrm{m})$ & 38.64 & 38.45 & 38.08 & $\downarrow$ \\
\hline IDI ( $\mu \mathrm{m})$ & 3.48 & 5.71 & 8.73 & 1 \\
\hline AvCID ( $\mu \mathrm{m})$ & 0.70 & 1.08 & 1.55 & $\boldsymbol{1}$ \\
\hline $\operatorname{AvUS}(\mathrm{N} / \mu \mathrm{m})$ & 0.59 & 0.59 & 0.59 & -- \\
\hline AvLS (N/ $\mu \mathrm{m})$ & 0.51 & 0.50 & 0.50 & -- \\
\hline AvED (N $\mu m)$ & 1.95 & 2.16 & 2.91 & $\boldsymbol{1}$ \\
\hline
\end{tabular}


Table 8. Computed RPI parameters as a function of maximum applied cyclic force.

\begin{tabular}{|c|c|c|c|c|}
\hline \multirow[b]{2}{*}{ RPI parameters } & \multicolumn{4}{|c|}{ Force } \\
\hline & $F=6 N$ & $F=8 N$ & $F=10 N$ & Trend \\
\hline ID1 ( $\mu \mathrm{m})$ & 26.32 & 32.46 & 37.97 & $\boldsymbol{1}$ \\
\hline 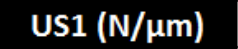 & 0.54 & 0.61 & 0.68 & $\boldsymbol{1}$ \\
\hline 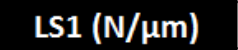 & 0.23 & 0.26 & 0.29 & $\boldsymbol{1}$ \\
\hline CID1 $(\mu \mathrm{m})$ & 4.27 & 5.02 & 5.69 & $\boldsymbol{1}$ \\
\hline TID $(\mu \mathrm{m})$ & 31.47 & 38.45 & 44.62 & $\boldsymbol{1}$ \\
\hline IDI ( $\mu \mathrm{m})$ & 4.86 & 5.71 & 6.44 & $\boldsymbol{1}$ \\
\hline $\operatorname{AvCID}(\mu \mathrm{m})$ & 0.94 & 1.08 & 1.25 & $\boldsymbol{1}$ \\
\hline AvUS (N/ $\mu \mathrm{m})$ & 0.51 & 0.59 & 0.66 & $\boldsymbol{1}$ \\
\hline 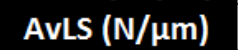 & 0.44 & 0.50 & 0.56 & 1 \\
\hline AvED $(\mathrm{N} \mu \mathrm{m})$ & 1.20 & 2.16 & 3.34 & $\boldsymbol{1}$ \\
\hline
\end{tabular}

Table 9. Computed RPI parameters as a function of indenter tip radius.

\begin{tabular}{|c|c|c|c|}
\hline \multirow{2}{*}{$\begin{array}{c}\text { RPI } \\
\text { parameters }\end{array}$} & \multicolumn{3}{|c|}{ Indenter tip radius } \\
\hline & Indenter tip radius $=15 \mu \mathrm{m}$ & Indenter tip radius $=\mathbf{3 0} \mu \mathrm{m}$ & Trend \\
\hline ID1 ( $\mu \mathrm{m})$ & 44.56 & 37.97 & $\downarrow$ \\
\hline 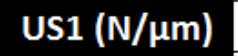 & 0.67 & 0.68 & $\sim 1$ \\
\hline LS1 (N/ $\mu \mathrm{m})$ & 0.30 & 0.29 & $\sim 1$ \\
\hline CID1 ( $\mu \mathrm{m})$ & 5.34 & 5.69 & $\boldsymbol{1}$ \\
\hline TID ( $\mu \mathrm{m})$ & 51.12 & 44.62 & $\downarrow$ \\
\hline IDI ( $\mu \mathrm{m})$ & 6.43 & 6.44 & $\sim \boldsymbol{1}$ \\
\hline AvCID ( $\mu \mathrm{m})$ & 1.19 & 1.25 & $\boldsymbol{1}$ \\
\hline AvUS $(\mathrm{N} / \mu \mathrm{m})$ & 0.66 & 0.66 & -- \\
\hline 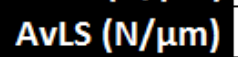 & 0.56 & 0.56 & -- \\
\hline AvED (N $\mu m)$ & 5.02 & 3.34 & 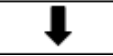 \\
\hline
\end{tabular}


Table 10. Computed RPI parameters as a function of number of cycles.

\begin{tabular}{|c|c|c|c|c|c|}
\hline \multirow{2}{*}{$\begin{array}{c}\text { RPI } \\
\text { parameters }\end{array}$} & \multicolumn{5}{|c|}{ No of cycles } \\
\hline & 2 cycles & 5 cycles & 7 cycles & 10 cycles & Trend \\
\hline ID1 ( $\mu \mathrm{m})$ & 37.97 & 37.97 & 37.97 & 37.97 & -- \\
\hline US1 ( $N / \mu m)$ & 0.68 & 0.68 & 0.68 & 0.68 & -- \\
\hline LS1 (N/ $/ \mathrm{m})$ & 0.29 & 0.29 & 0.29 & 0.29 & -- \\
\hline CID1 ( $\mu \mathrm{m})$ & 5.69 & 5.69 & 5.69 & 5.69 & -- \\
\hline TID ( $\mu \mathrm{m})$ & 44.60 & 44.62 & 44.62 & 44.62 & $\sim 1$ \\
\hline AvCID $(\mu \mathrm{m})$ & 2.99 & 1.25 & 0.90 & 0.64 & $\nabla$ \\
\hline AvUS $(N / \mu \mathrm{m})$ & 0.66 & 0.66 & 0.66 & 0.67 & $\sim \downarrow$ \\
\hline AvLS (N/ $\mu \mathrm{m})$ & 0.45 & 0.56 & 0.59 & 0.62 & 1 \\
\hline AvED (N $\mu m)$ & - & 3.34 & 3.14 & 2.96 & $\nabla$ \\
\hline
\end{tabular}

Table 11. Comparison between RPI experimental results (Granke et al. 2014) and computed RPI results. (r: indenter tip radius).

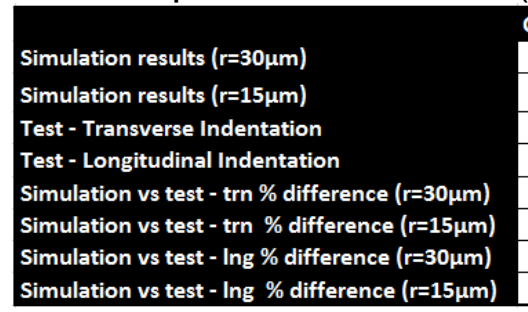

\begin{tabular}{|c|c|c|c|c|c|}
\hline CID1 $(\boldsymbol{\mu m})$ mid-range LS1 $(\mathbf{N} / \boldsymbol{\mu m})$ mid-range US1 $(\mathbf{N} / \boldsymbol{\mu m})$ mid-range & AvCID $(\boldsymbol{\mu m})$ & \multicolumn{2}{c|}{ AvLS $(\mathbf{N} / \boldsymbol{\mu m})$} & \multicolumn{1}{c|}{ AvUS $(\mathbf{N} / \boldsymbol{\mu m})$} \\
\hline 5.69 & 0.29 & 0.68 & 1.25 & 0.56 & 0.66 \\
\hline 5.34 & 0.30 & 0.67 & 1.19 & 0.56 & 0.66 \\
\hline 6.1 & 0.21 & 0.61 & 1.565 & 0.476 & 0.66 \\
\hline 4.7 & 0.23 & 0.7 & 1.24 & 0.749 & 0.724 \\
\hline$-6.72 \%$ & $38.10 \%$ & $11.48 \%$ & $-20.13 \%$ & $17.65 \%$ & $0.00 \%$ \\
\hline$-12.43 \%$ & $41.94 \%$ & $10.51 \%$ & $-24.16 \%$ & $17.10 \%$ & $-0.53 \%$ \\
\hline $21.06 \%$ & $26.09 \%$ & $-2.86 \%$ & $0.81 \%$ & $-25.23 \%$ & $-8.84 \%$ \\
\hline $13.65 \%$ & $29.60 \%$ & $-3.70 \%$ & $-4.28 \%$ & $-25.58 \%$ & $-9.32 \%$ \\
\hline
\end{tabular}


Figures:

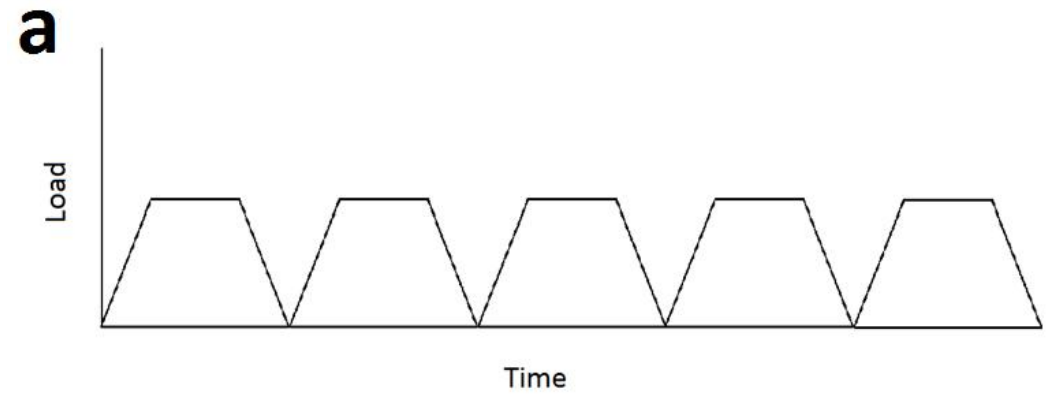

b

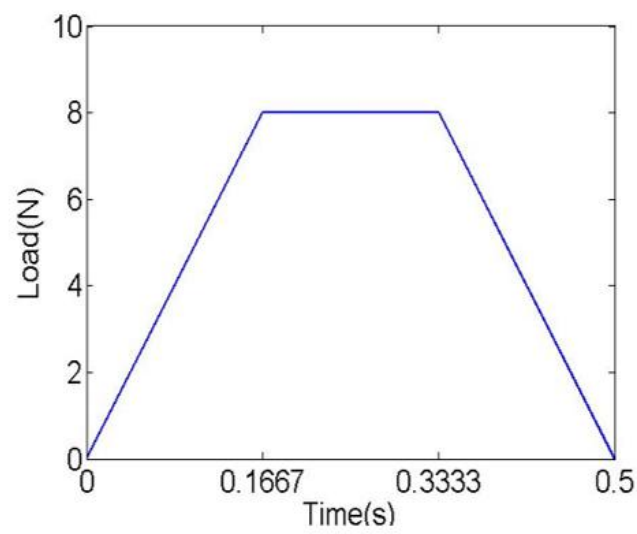

Figure 1. Reference point indentation cyclic loading. a) Typical 5 cycles of RPI

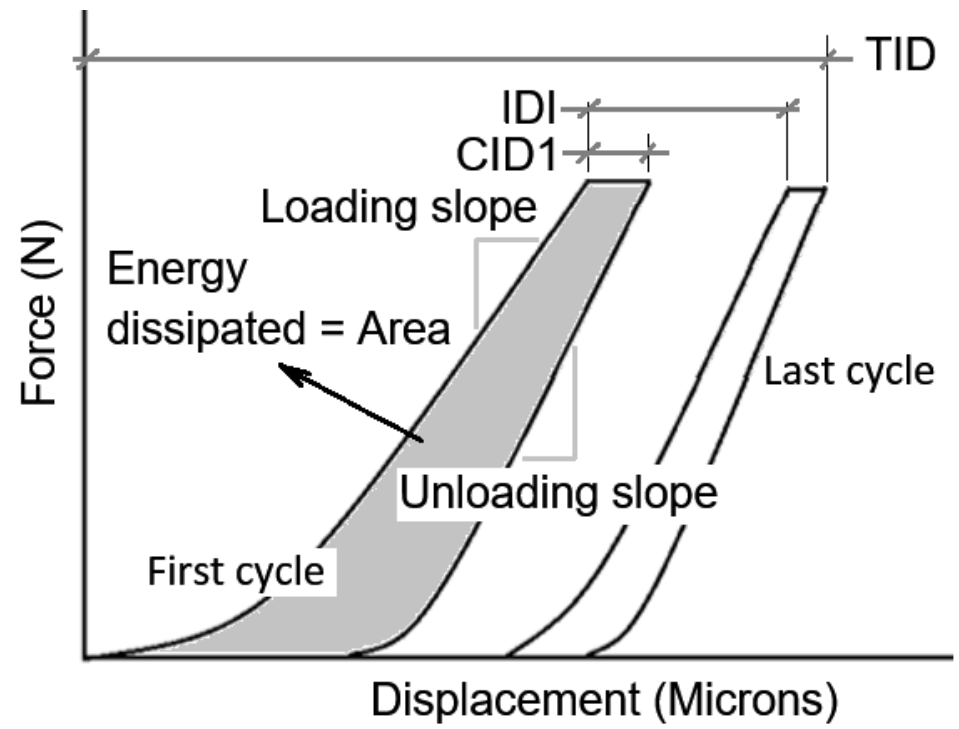

Figure 2. Typical force vs displacement curves for RPI first and last loading cycles 


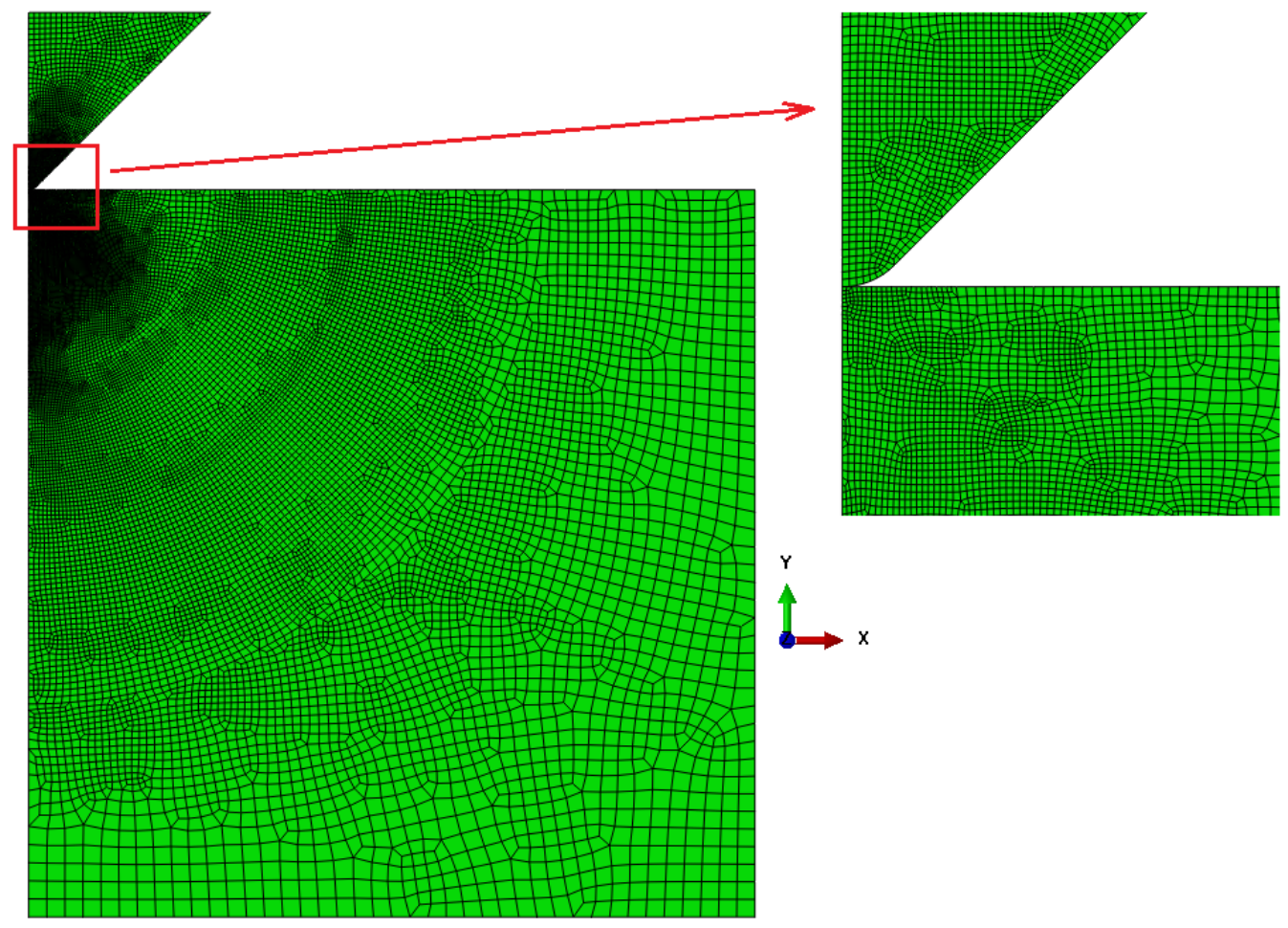

Figure 3. Axisymmetric finite element model of reference point indentation. 

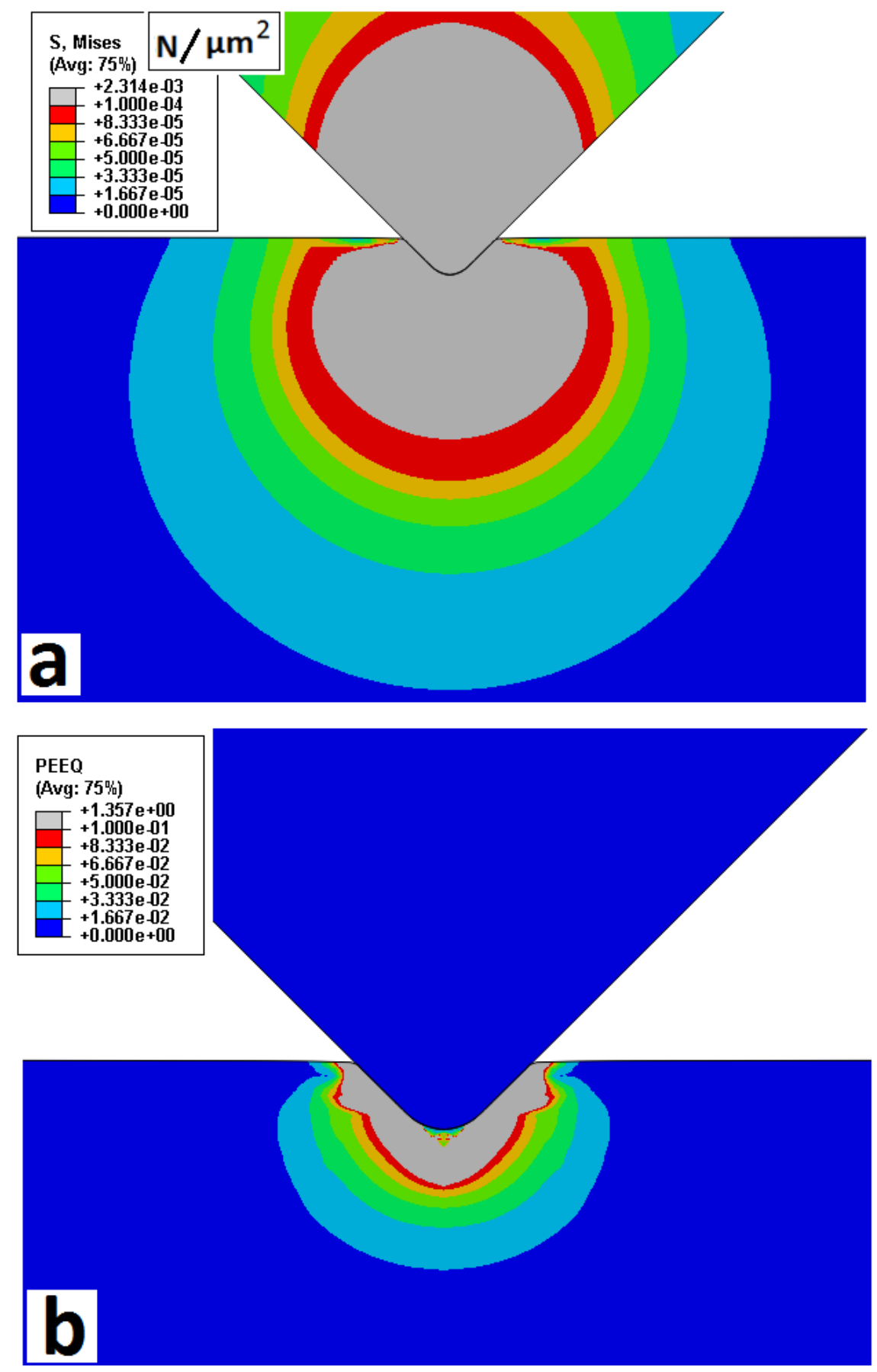

Figure 4. RPI simulation results at the end of the $5^{\text {th }}$ loading cycle using material properties as given in Tables 2 and 3 in bold font. a) Von Mises stress contours. b) Equivalent plastic strain contours. 

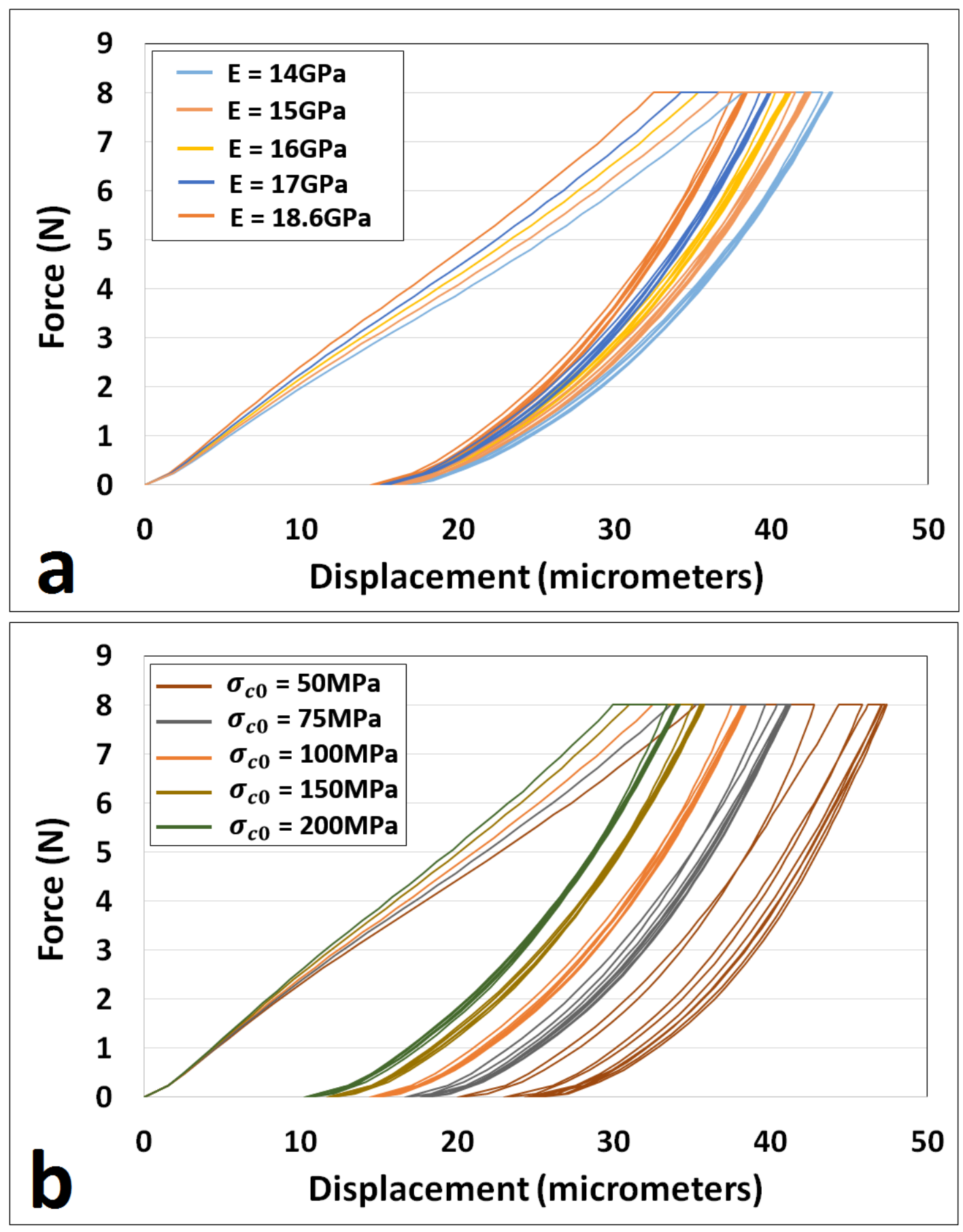

Figure 5. RPI simulations results: a) Young's modulus effect. b) Compressive yield stress effect. 

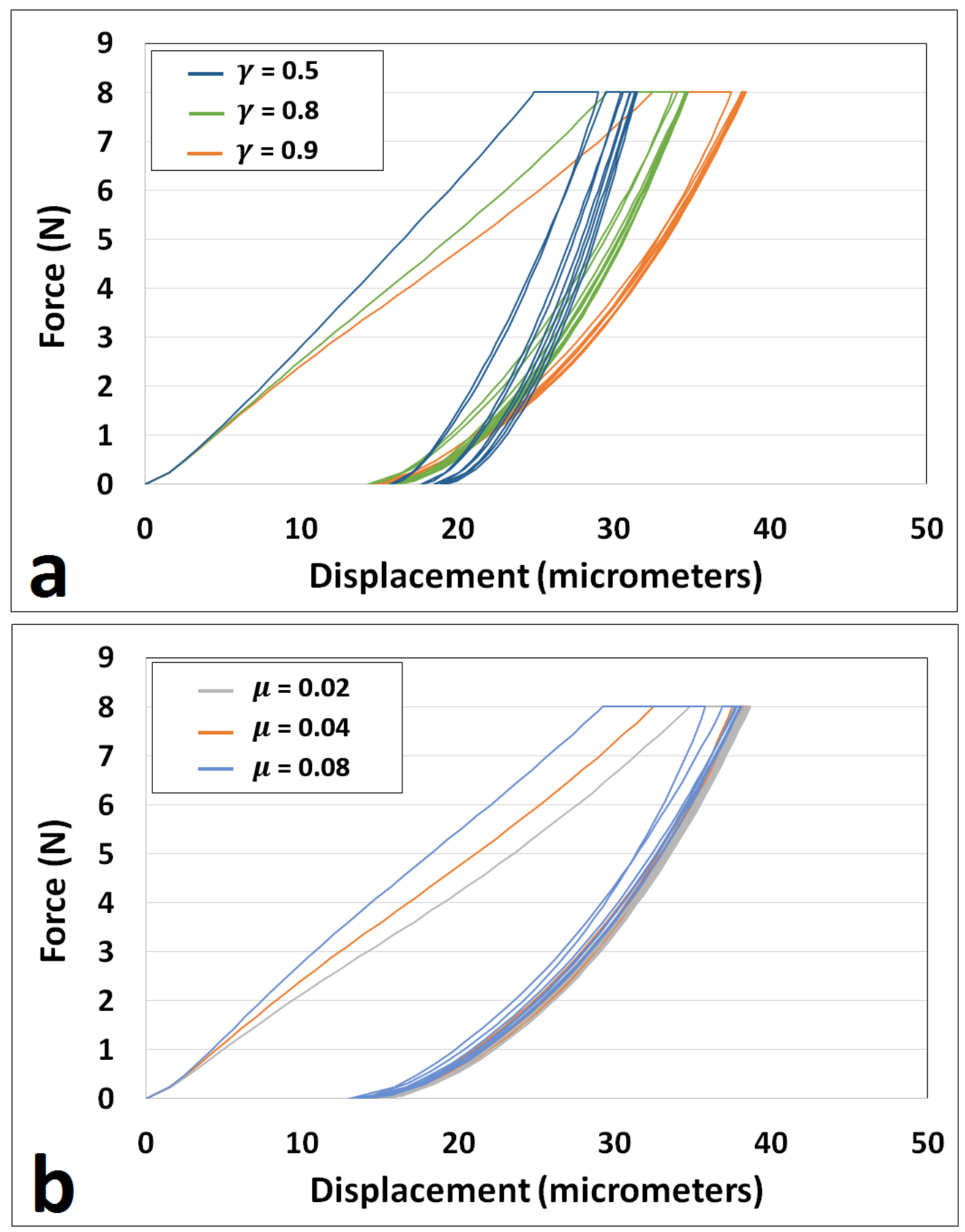

Figure 6. RPI simulations results: a) Damage constant effect. b) Viscosity constant effect. 

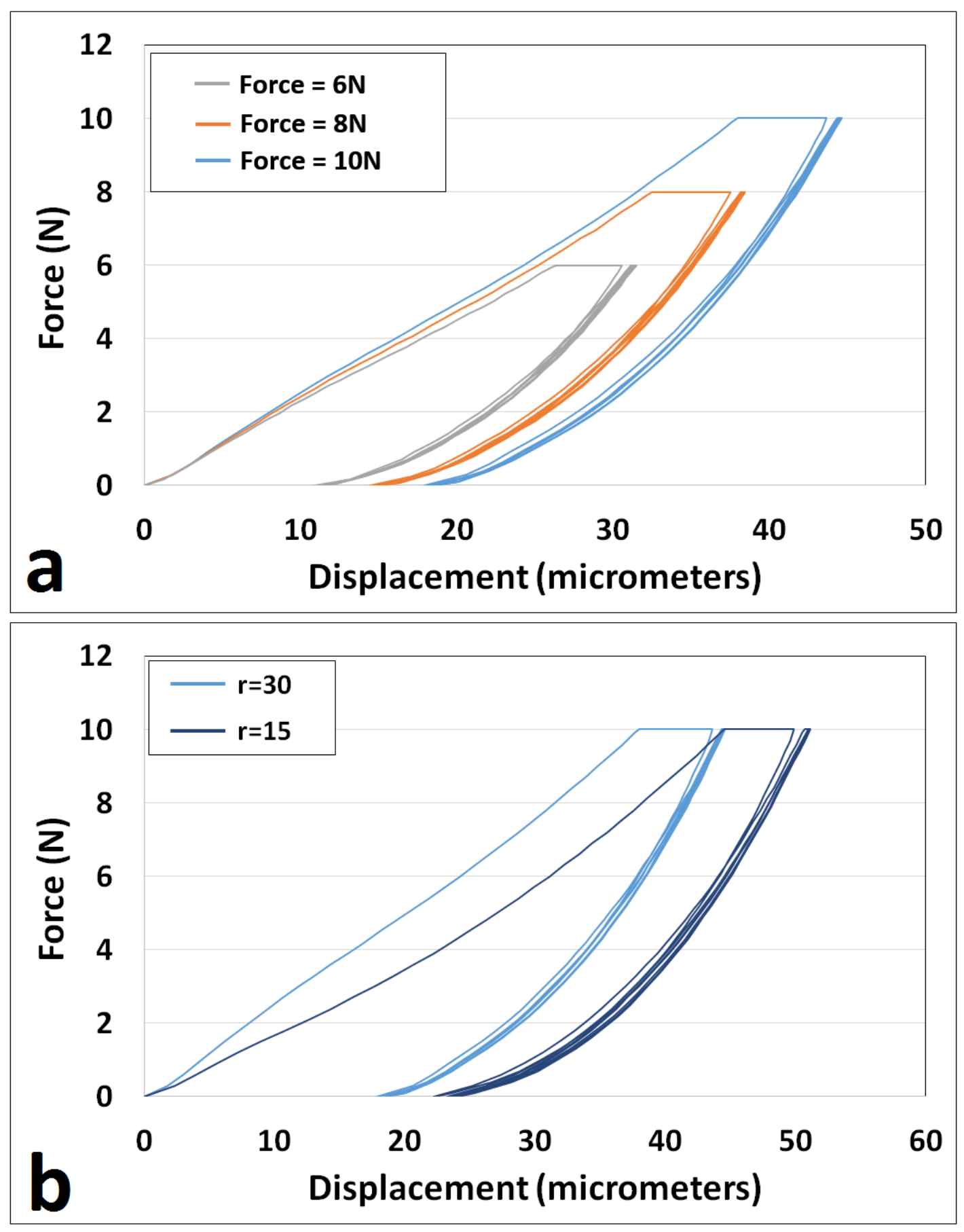

Figure 7. RPI simulations results: a) Applied peak cyclic force effect. b) Indenter tip radius effect. 


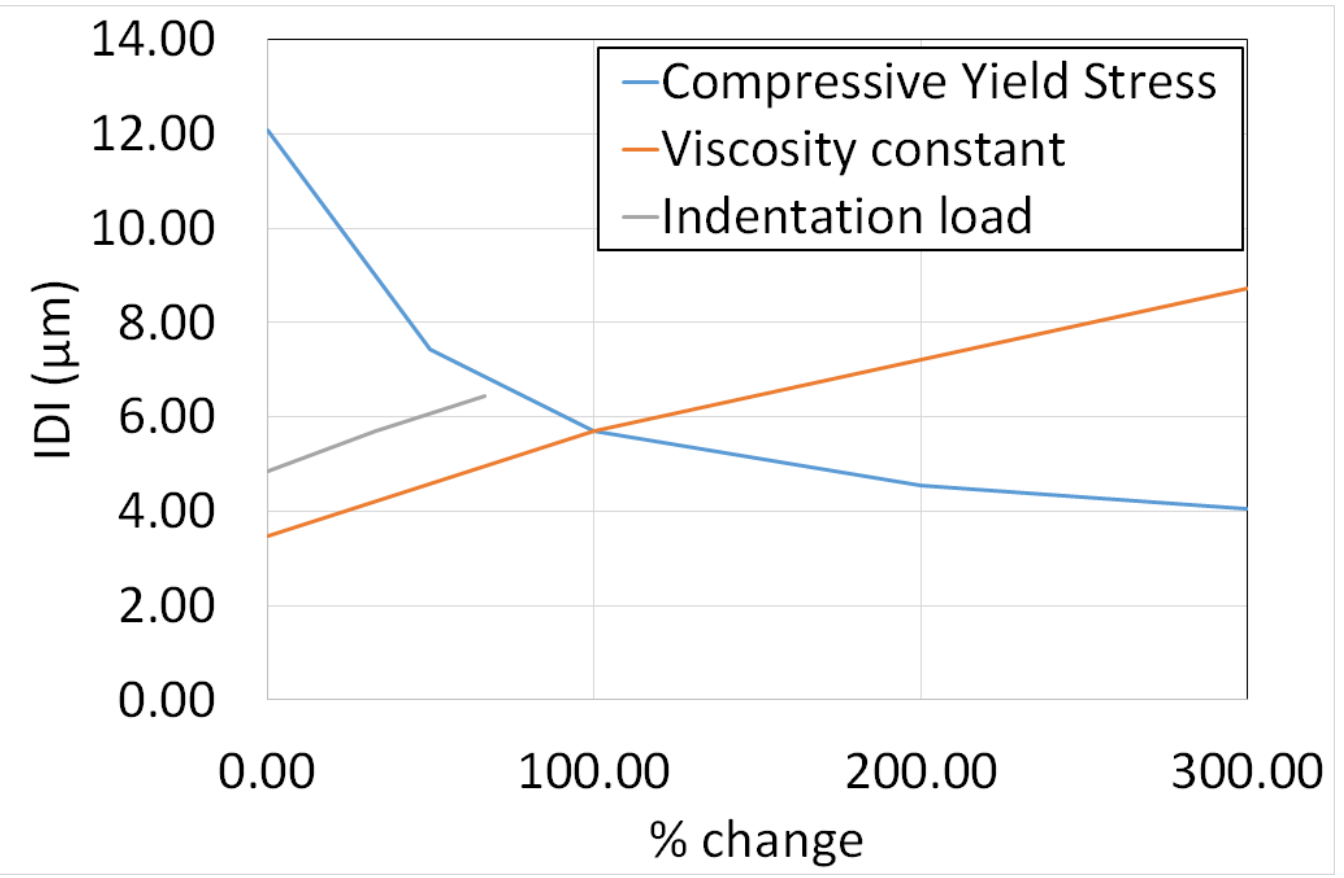

Figure 8. IDI relation to compressive yield stress, viscosity constant, and indentation load value.

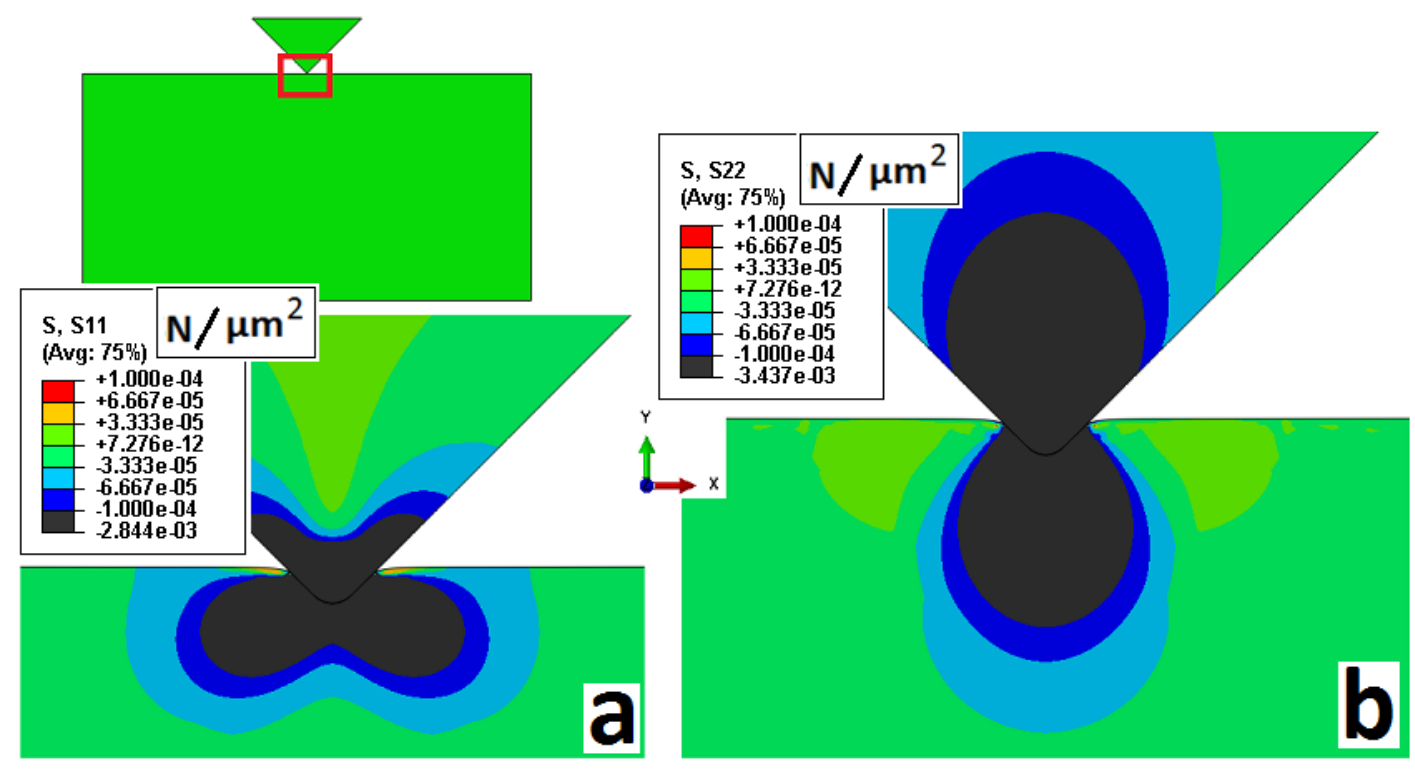

Figure 9. Stress contours at the end of $1^{\text {st }}$ cycle loading using material properties as given in Tables 2 and 3 in bold font: a) $\sigma_{11}$ and b) $\sigma_{22}$. The contours shown in the image are mainly in a dark color (black), which represents compressive stresses. Tensile stresses are represented mainly in green. The tensile stresses are many orders smaller compared to the compressive stresses, 31 as shown in the figure. 